nent soit-il, sont-ils comparables au choix méthodique et sagace d'une commission de classement? )

" Nommer au concours des infirmières, même militaires, apparait aux yeux les moins prévenus comme un danger, comme une source d'insuccès pour une institution aussi utile que désirable; car celte formule surannée de sélection risque de fausser, dans son mécanisme même, l'œuvre nouvelle si sagement entreprise et múrie par le Ministre de la guerre."

Sans vouloir émettre un avis quelconque dans un débal qui ne nous concerne pas, nous croyons savoir que l'opinion émise ici par le $D^{r}$ Letulle, s'est trouvée confirmée ailleurs par des expériences vécues; du reste, pour tout ce qui concerne une activité professionnelle, qui doit nécessairement être beaucoup plus prati. que que théorique, qui doit être une expérience plus qu'une science, l'épreuve du stage pratique prolongé vaudra toujours mieux que le plus brillant concours; celui-ci pourrait du reste plutôt être réservé pour l'admission à celui-là.

Quoi qu'il en soit, nous saluons avec joie la rentrée des femmes comme infirmières militaires dans l'armée où les gardes-malades des sociétés de secours de la Croix-Rouge les ont déjả précédées dès longtemps à la satisfaction de tous.

Dr Ferrière.

\title{
CHIENS SANITAIRES ET CHIENS DE TRANSPORT
}

Un concours international de dressage de chiens sanitaires a réuni, en janvier dernier, à Paris, devant une assistance nombreuse et en présence de délégués du Ministère de la guerre et de la Société de secours aux blessés militaires, un cerlain nombre de chiens dressés pour la recherche des blessés. Le résultat de ce premier concours a été fort intéressant et l'on peut dès maintenant envisager comme nettement efficace le concours de cet intelligent animal sur le champ de bataille.

Ont pris part au concours des chiens des types berger, airedale et dobermann, dressés en Allemagne, en Suisse et en France. Le 
jury était composé de MM.le capitaine de Stephanitz (Allemagne), Otto Rahm (Suisse) et le capitaine Tolet (France).

Le programme de la recherche des blessés était ainsi rédigé :

" Le chien, accompagné de son maître, battra le terrain à 150 ou 200 mètres et devra retrouver le blessé dans le moins de temps possible. L'ayant trouvé, aboyer pour prévenir son maître, ou, ayant trouvé le blessé, ne pas aboyer et rapporter à son maitre une pièce d'équipement, képi ou autre. Le chien pourra également revenir vers le maitre et aboyer quand il sera près de lui, conduire ensuite son maitre vers l'homme. Toutes ces méthodes sont admises. Le nombre des blessés ne sera pas supérieur à dix.

C'est un chien suisse, appartenant à M. Hauri de Lenzburg, qui a remporté le premier prix. Il faut observer toutefois que c'est un chien français, hors concours parce qu'il avait été dressé par le capitaine Tolet, fonctionnant comme juge, qui, de l'avis de to u les spectateurs, a été supérieur de beaucoup à tous les autres concurrents et aurait, de ce fait, remporté le premier prix.

Dans un article du Caducée ${ }^{1}$ M. Granjux apprécie comme suit le résultat de ce concours :

- De ce que nous avons vu, il ressort pour nous que le dressage français des chiens sanitaires est supérieur à celni employé par les Suisses ou les Allemands. Cela tient, croyons-nous, à ce que les chiens français avaient été dressés exclusivement comme chiens sanitaires et qu'on leur avait appris à battre le terrain.

« Au contraire les chiens allemands ou suisses ne sont chiens sanitaires que par ricochet; ils ont surtout été dressés comme chiens de police, c'est-à-dire habitués à suivre une piste sur laquelle on les met. En conséquence, tandis que le chien français livré à lui-même se mettait immédiatement à quêter et trouvait, sans aide aucune, les blessés, les dresseurs allemands ou suisses étaient parfois obligés d'aider de la parole ou du geste leurs animaux. "

Cette observation confirme la remarque qui terminait notre précédent article sur ce sujet ${ }^{2}$, constatant l'incompatibilité qui existe entre le chien policier et le chien sanitaire; il semble qu'il sera fort difficile d'obtenir de l'animal une différence de procédé

$1 N^{\circ} 2,1908$, p. 21.

2 Bulletin $\mathrm{n}^{0} 153$, p. 42. 
suivant qu'il s'agira d'un blessé à secourir ou d'un malfaiteur à traquer et que l'éducation du chien risque fort de souffrir de la poursuite de ce double but; il est non moins vrai, malheureusement, que l'éducation laborieuse de chiens uniquement sanitaires, à des fins plus ou moins platoniques, - car combien de générations de chiens sanitaires pourront se suivre sans avoir jamais eu l'occasion de travailler effectivement sur le champ de bataille, - risque d'être décourageante à la longue pour les éleveurs et d'aboutir en fin de compte, à l'échec d'une entreprise aussi intéressante. Nous en conclurions volontiers, pour autant que nous sommes placés pour émettre une opinion dans une question qui nous est étrangère, que la question reste ouverte quant au mode d'éducation du chien policier ou sanitaire, malgré l'incontestable justesse du point de vue français dans cette question.

Voici encore, d'autre part, une nouvelle application de l'emploi du chien dans les services de santé ; il s'agit du chien de trait pour le transport des blessés.

* En parcourant la théorie de l'école de l'infirmier et du brancardier mlitaires, j'ai pensé, dit M. le lieutenant Puisais dans un mémoire sur ce sujet ${ }^{1}$, que l'on pourrait remplacer avantageusement le brancard roulant, trainé par deux hommes, par une petite voiture brancard, légère, à quatre roues et traînée par un chien. Et l'auteur décrit une ingénieuse voiturette pliable, à rones légères, avec roulement à billes et bien suspendue, sur laquelle vient se placer le brancard règlementaire.

Pour l'usage sur le champ de bataille, une voiture brancard dépliée et attelée de son chien, transporte trois autres voitures pliées et trois brancards ordinaires. « Cette voiture, dit M. Puisais, reste sous la garde d'un infirmier sur le chemin le plus rapproché de la partie du champ de bataille à explorer ; cet infirmier déplie les voitures. Trois chiens disponibles, porteurs de leur harnachement, de deux bidons remplis d'eau et de quelques objets pour pansements sommaires vont, avec les infirmiers porteurs de brancards, à la recherche des blessés. Ceux-ci, placés sur les brancards vont rejoindre l'endroit où se trouvent les voitures et le chargement est effectué. Quand plusieurs blessés sont prêts à être transportés, les voitures, sous la conduite de deux infirmiers à bicy-

\footnotetext{
${ }^{1}$ Archives de médecine et de pharmacie militaires, $\mathrm{n}^{\circ} 1,1908$, p. 72.
} 
- clette, -sont dirigés sur le point fixé par le docteur : infirmerie ou: poste d'évacuation.

L'avantage de ce procédé réside surtout dans l'économie du personnel. Deux infirmiers à bicyclette pourront, pense M. Puisais, accompagner quatre à six voitures attelées, tandis qu'actuellement il faut deux intirmiers pour traîner un brancard roulant; il resterait ainsi six ou huit infirmiers disponibles pour la recherche des blessés ou pour tout autre service. Le transport, du même fait, sera plus rapide; M. Puisais estime que les chiens l'effectueront à raison de 10 kilomètres environ à l'heure. Le montage ou démontage du chariot-brancard peut être effectué par un seul homme en quelques minutes et les chiens ne sont, d'autre part, pas d'un entretien difficile. Inutile d'ajouter qu'il faut, pour ce mode de transport, des chiens de grande taille et robustes, ainsi que de bonnes routes avec des pentes modérées.

Il sera intéressant de voir réaliser, par des essais systématiques, sur un terrain de manœuvres, le projet sans doute fort ingénieux de M. Puisais, car cette nouvelle utilisation des chiens sanitaires serait la bienvenue sur le champ de bataille où les brancardiers sont toujours insuffisants en nombre et d'un recrutement si difficile.

Mentionnerons-nous ici, à propos de ces intéressants et nouveaux auxiliaires des services de santé, la diatribe sévère de $\mathbf{M}$. le 'médecin-major, $\mathrm{D}^{\mathrm{r}}$ Bonnette, contre des chiens de quartier? ${ }^{1}$ Dans beaucoup de casernes et d'hôpitaux militaires des chiens, plus ou moins nombreux, plus ou moins sans maître aussi, en quête d'une caresse ou d'un morceau de pain, caressés, choyés, dorlotés par les hommes, deviennent, pense l'auteur, un danger constant pour la troupe en constituant un moyen fréquent de transmission des maladies infectieuses.

Au nom de l'hygiène, il en demande la suppression, de même du reste que celle des femmes et des enfants des ménages militaires. " La caserne, observe-t-il, doit être aux soldats et rien qu'aux soldats. "Sans doute! en principe; mais le microbe de l'ennui, de l'invincible ennui résultant d'une existence dénuée par trop des légitimes et modestes distractions de la sociabilité, même avec de simples animaux domestiques, ne risque-t-il pas d'entrâ̂-

\footnotetext{
${ }^{1}$ Caducée, 1908, n 1, p. 3.
} 
ner les hommes à aller chercher, dans leurs moments de loisir, des germes infectieux plus dangereux encore, ailleurs?

Nous ne songeons pas à prendre la défense des chiens de quartier, loin de là, mais le principe de la «caserne rien qu'aux soldats» ne saurait non plus être poussé trop loin sans quelque danger.

Dr Ferrière.

\section{GRANDE-BRETAGHE}

LIEUT.-GOL. W.-G. MACPHERSON. - DU RÔLE DES SOCIÉTÉS DE LA. CROIX-ROUGE EN TEMPS DE PAIX ET EN TEMPS DE GUERRE

M. le lieut.-col. Macpherson, dont le nom est bien connu dans les sphères de la Croix-Rouge, et qui fut notamment un des délégués de la Grande-Bretagne à la Cionférence de revision de 1906 , a présenté le 6 février dernier à la Royal United Service Institution une communication sur le rôle des sociétés de la Croix-Rouge en temps de paix et en temps de guerre, en prenant pour point de départ de son exposé la situation faite à ces dernières par la Convention de Genève revisée, du 6 juillet 1906.

L'auteur voit la légitimation du privilège ainsi accordé à ces sociétés dans le travail accompli par elles aux còtés du service sanitaire officiel. L'affirmation que l'on rencontre dans cerlaines bouches que les sociétés ne doivent pas être chargées de soins qui

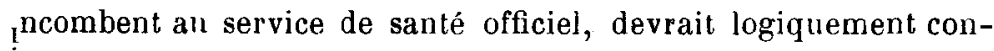
duire à la suppression de ces sociétés. Elles servent à canaliser et utiliser pratiquement ce flot d'humanitarisme qui se manifeste autour des blessés et des malades. Elles ont su profiter des bonnes volontés et des capacités qui se marifestent en dehors des cadres de l'armée, notamment dans le sexe féminin, en faveur des victi. mes de la guerre. L'auteur montre par un bref aperçu de l'organi sation des Croix-Rouges dans les différents pays comment cette tâche a été réalisée, depuis les sociétés entièrement libres de toute 